\title{
LOS SIG: NUEVAS HERRAMIENTAS PARA EL ESTUDIO DE LOS ESPACIOS PALEOLÍTICOS
}

\author{
SIG: new tools for the study of Paleolithic space
}

\author{
Paula ORTEGA MARTÍNEZ* \\ E-mail: ortegap@usal.es \\ Universidad de Salamanca
}

Fecha de recepción: 20-01-2010

Fecha de aceptación: 29-01-2010

RESUMEN: El hombre, como individuo y como grupo, tiene una concepción del lugar que habita. Este enfoque, permite interpretar la forma de vida de las sociedades en contextos espaciales significativos. No se puede aislar las sociedades paleolíticas del espacio que dominaban y del que se apropian para el desarrollo de sus labores cotidianas. Los suelos de habitación constituyen un testimonio latente de las formas de vida del hombre paleolítico, del que pocos datos se conservan, salvo aquellos que se derivan de la excavación de yacimientos y del estudio del arte conservado, ambos reflejo de las actividades desarrolladas en el espacio doméstico.

Los patrones habitacionales tienen que ser comprendidos en términos de relaciones espaciales que los materiales entablan entre si, en un marco genérico, el nivel de ocupación. Para este estudio contamos con los Sistemas de Información Geográfica, herramientas indispensables para la gestión de los datos principalmente espaciales, pero también funcionales o tecnológicos.

Palabras Clave: espacio, hábitat, Paleolítico Superior, SIG, metodología, suelos de habitación, suelos de ocupación

ABSTRACT: The man, as an individual and as a group, has a dwelling place conception. This approach, allows to interpret the lifestyles of societies in spatial significant contexts. It is not possible to isolate paleolithic societies of the space they dominated and they occupied in order to develop their daily tasks. Dwelling floors constitute a latent testimony of 
the lifestyles of paleolithic societies, from which little information remain, except those stemmed from the excavation of deposits and from the study of the preserved art, both of them reflect the activities developed in the domestic space.

Habitat patterns have to be understood in terms of spatial relations that the materials have among themselves, in a generic frame, the occupation levels. The Systems of Geographical Information, are indispensable tools for the management of spatial information primarily, but also functional or technological.

Keywords: Space, Hábitat, Intra-site, Upper Paleolithic, GIS, Metodology, Dwelling floors Occupation floors

\section{INTRODUCCIÓN}

El presente trabajo pretende realizar un acercamiento al estudio del espacio habitado por los grupos humanos del Paleolítico Superior a través de la aplicación de nuevas tecnologías como son los Sistemas de Información Geográfica. Estas herramientas, al alcance de los prehistoriadores, facilitan el estudio, análisis y comprensión de la organización del espacio dentro de una zona de hábitat que se ha conservado en la forma de los distintos suelos de ocupación. Planteamos las posibilidades metodológicas que estas herramientas nos ofrecen, de manera que nos permiten analizar y responder a cuestiones concretas y complejas.

\section{ESPACIO PALEOLÍTICO}

\section{EL ESPACIO Y LOS SUELOS DE OCUPACIÓN}

El espacio, ha sido conceptualizado y estudiado desde distintas perspectivas a lo largo de la historia. Entendido no sólo desde una perspectiva absoluta, en la que ya los filósofos atomistas griegos lo definían como marco contenedor en el que se encuadran todos los objetos; sino también desde una perspectiva relativa entendida como una relación entre cosas, una cualidad posicional, que se separa de la tradición newtoniana con la llegada de Leibniz ${ }^{1}$ y posteriormente la teoría de la Relatividad ${ }^{2}$. Es en la primera mitad del s. XX cuando nació una nueva orientación basada en la concepción del espacio sensitivo como una categoría, es en este momento cuando entra en la ecuación el ser humano que se relaciona con el entorno ${ }^{3}$.

El espacio, desde nuestro punto de vista, es un concepto indisoluble con el hombre, ya que este tiene una propia concepción del espacio en la 
medida en que constituye el marco en el que desarrolla su vida. No podemos entender las dinámicas sociales de los grupos paleolíticos aisladas del espacio que habitaban, ocupaban y dominaban y del que se apropiaban para desarrollar sus labores cotidianas.

Pocas son las fuentes que nos permiten comprender el comportamiento de los grupos humanos del Paleolítico en el espacio que ocupaban. Tan sólo contamos con el registro arqueológico y gráfico para comprender como se articulaban estas sociedades en el espacio en el que estaban inmersos.

El concepto de suelos de ocupación fue definido ya en 1975 por LeroiGourhan, quien afirmaba que el suelo de habitación es una "superficie reconocible sobre la cual ha vivido el hombre paleolitico durante un lapsus de tiempo suficientemente corto para que podamos esperar deducir de la posición de los vestigios algo que sugiere las actividades". Rigaud, en la misma línea, lo define como el "resultado intacto o casi intacto de la ocupación de un sitio por un grupo bumano durante un cierto periodo de tiempo"s.

Los suelos de habitación son pues un testimonio latente de las formas de vida del hombre para unos períodos, concretamente el Paleolítico Superior, en los que pocos datos se conservan, salvo los que se derivan de la excavación de yacimientos y del estudio del arte conservado. Son, por tanto, el conjunto de todos los restos materiales que se depositan en la superficie del área en la que se desarrolla la vida cotidiana. Es por este motivo, que las estructuraciones del espacio paleolítico se conciben en términos de relaciones espaciales, como fenómenos de acumulación y dispersión, que los materiales entablan entre si, en el marco genérico que constituye el nivel de ocupación, por lo que tienen que ser comprendidas de una manera dinámica.

El suelo, como las paredes decoradas de las cuevas, constituyen un reflejo de las actividades desarrolladas en el espacio doméstico. Tan sólo a través de la restitución de toda la información recabada en el transcurso de las excavaciones podremos reconstruir los patrones habitacionales de las sociedades paleolíticas.

Para esto contamos con la inestimable ayuda de los Sistemas de Información Geográfica, herramientas indispensables para la gestión de los datos espaciales, pero no sólo espaciales, sino también funcionales o tecnológicos, recuperados en el transcurso del proceso de excavación, incluso en yacimientos excavados antiguamente.

Entendemos como necesarios el cotejo de los resultados obtenidos con otro tipo de datos, tales como los que nos pueden aportar las investigaciones etnoarqueológicas, que ya se han documentado en muchas investigaciones sobre los suelos de ocupación ${ }^{6}$, o incluso la propia experimentación. 
Se plantea, por último, la posibilidad de estudiar experimentalmente algunos procesos específicos, como pudieran ser los propios procesos de talla, que nos permite la reproducción de la labor en el espacio, de manera que se controlen las variables tales como los gestos, el tiempo, la materia, etc.

\section{HisTORIOGRAFÍA}

La investigación de los patrones habitacionales latentes en el registro arqueológico no ha sido un elemento integrante en las investigaciones de las sociedades paleolíticas hasta mediados del s XX. Esto no quiere decir que no contemos con estudios pioneros sobre el tema, como son los desarrollados por Peyrony a principios del s. XX en Forneau du Diable ${ }^{7}$ o de P.P. Efimenko en Kostienki ${ }^{8}$ Pero no es hasta mediados de siglo, cuando se produce una saturación en el ámbito de las investigaciones paleolíticas, que se basaban en estudios tipológicos de los conjuntos paleolíticos, lo que unido a los avances en los sistemas de datación, los estudios estratigráficos y geomorfológicos, hace que se produzca una necesidad de nuevos focos de investigación, produciéndose así una diversificación de los temas con los que se intentará conocer/definir las sociedades que nos ocupan.

La Prehistoria no puede reducir sus ambiciones a los inventarios de útiles y fósiles. Estos inventarios no son más que un esqueleto, que unido a los análisis sedimentológicos y palinológicos, permitirá establecer una cronología, base indispensable de todo estudio más pausado, pero insuficiente para reconstruir la vida prehistórica?.

El nacimiento de los estudios de arqueología de los suelos, que en un primer momentos se basó en el simple levantamiento planimétrico de los mismos, se puede decir que viene ligado al descubrimiento del yacimiento de Pincevent y los estudios espaciales llevados a cabo en el mismo. Sin duda, estos estudios espaciales son producto de un largo desarrollo metodológico que el equipo de investigadores de este proyecto ya había iniciado en yacimientos de las cuevas d'Arcy-sur Cure (Yonne). Aunque ya se habían encontrado yacimientos semejantes en el centro y el este de Europa, Rusia y Ucrania, las características de este yacimiento han hecho que se convierta en un referente mundial.

Fue Leroi-Gourhan quien, analizando la habitación 36 de Pincevent, propuso la primera interpretación sobre el aprovechamiento del espacio doméstico "modelo teórico de Pincevent" empírico surgido de las observaciones de los restos hallados en el yacimiento de Pincevent, lo que hace que sea muy específico para este yacimiento. No es, por tanto, un modelo teórico que pudiera ser contrastado con otras rea- 
lidades arqueológicas. Se basa, pues, en la repetición de elementos como hogares, desechos de talla o restos óseos.

Este modelo se erigió como un importante avance en las investigaciones paleolíticas, pues en el momento en el que se creó, poco o nada se estaba investigando en esta vía. Leroi-Gourhan no se limitó a analizar las concentraciones o dispersiones de material, sino que, a la vez, las interpretó desde una perspectiva funcional.

Sin embargo, la proliferación de los descubrimientos de hábitats intactos y las investigaciones en esta línea demostraron lo poco generalista del modelo, aspecto muy criticado por investigaciones como la de L.R. Bindfod ${ }^{11}$ quien, debido a su formación de etnoarqueólogo, discute la validez del modelo de Leroi-Gourhan y propone otras alternativas, si cabe más generalistas. Este debate abierto entre las dos formas de estudiar la prehistoria no se cerrará hasta años después, cuando los discípulos de ambos investigadores hallen una postura conciliadora entre ambos enfoques, asumiendo la validez de ambas interpretaciones, de manera que se entiendan como complementarias.

En medio de la gran vorágine cientificista que vivieron las investigaciones de las ciencias sociales en los años 70 , se creó la necesidad de buscar nuevos enfoques para el estudio del período cronológico que nos ocupa, tales como la socioeconomía y la paleoeconomía. Nació un nuevo método de análisis arqueológico en el que se trataba de obtener teorías económicas a partir no sólo de los artefactos sino también de las posibilidades que ofrecían los asentamientos en si.

Se creó entonces la "escuela paleoeconómica de Cambridge" de la mano de Clarke ${ }^{12}$ Hodder y Orton ${ }^{13}$ que bebía directamente de esta nueva tendencia historiográfica. El método de análisis espacial en Arqueología es introducido en Europa de la mano de Vita Finzi y Higgs.

En las últimas décadas, la aplicación de nuevas tecnologías y, concretamente, las más recientes técnicas estadísticas y gráficas, hicieron avanzar los análisis espaciales más allá de las prácticas descriptivas. Los avances informáticos permitieron una aceleración de las técnicas cuantitativas, no sin añadir ciertos problemas. La facilidad para obtener resultados óptimos, las nuevas tecnologías, las reconstrucciones $2 \mathrm{D}$ y $3 \mathrm{D}$ y la realidad virtual hacen que corramos el riesgo de aplicar estas tecnologías sin un trasfondo teóricometodológico, que estas mismas tecnologías poseen, y sin duda es importante y necesario. Así, como dice R. Ontañón ${ }^{14}$, estamos ante una situación en la que se denota una carencia en una aparato teórico y metodológico para los estudios de suelos y estructuras de habitación, que hasta ahora se basaban en razonamientos intuitivos, en ocasiones apoyados por estudios etnográficos o experimentales debido a la "inmadurez de esta especialidad disciplinaria" lo que dificulta el desarrollo del conocimiento global. 
La aplicación de modelos teóricos de disposición del espacio en época paleolítica se ha basado principalmente en yacimientos al aire libre. Esto viene motivado por las condiciones de conservación de este tipo de yacimientos, por norma general más intactos. Tenemos que tener en cuenta que nuestro estudio se basa en estructuras y artefactos depositados o desechados por el hombre paleolítico, que posteriormente han sido agregados/asimilados a una estructura de formación geológica, que es la propia estratigrafía del lugar. Así pues, debemos tener en cuenta el hecho de que los yacimientos arqueológicos al aire libre presenten una estratigrafía más sencilla, en la que intervienen procesos deposicionales de menor carga erosiva, que puedan producir el traslado de los artefactos ${ }^{15}$.

En cambio, los yacimientos en cueva sufren más movimientos deposicionales, debido a la propia vida de la misma. Sin embargo las nuevas tecnologías al alcance del investigador hacen que en algunos casos estas dificultades puedan ser salvadas, llegando a conclusiones importantes sobre el hábitat en cueva. Es por ello que no debemos descartar estos contextos del análisis espacial de manera categórica, sino que debemos ser críticos con la calidad de los datos que tenemos.

\section{ESTUdio DE LOS SUELOS PALEOLÍTICOS}

\section{Problemática CON EXCAVACIONES ANTIGUA}

En los últimos años han proliferado los análisis espaciales en el ámbito del estudio de las sociedades paleolíticas. Sin embargo, estos estudios han sido en su mayoría análisis complementarios e investigaciones específicas sobre determinados elementos del registro arqueológico. Esto ha producido una gran cantidad de estudios espaciales parciales en los que se estudian diferentes ámbitos del registro arqueológico y se le dota, a cada elemento, de atributos espaciales. Esto impide conocer la disposición y relación espacial de materiales de distinta índole, y nos imposibilita el crear hipótesis globales sobre el comportamiento de las sociedades en las zonas de hábitat.

Las nuevas tecnologías al alcance de la arqueología han favorecido, en las últimas décadas, la realización de registros más exhaustivos de los levantamientos planimétricos. La recuperación de todos los elementos del sustrato con sus debidas referencias espaciales favorece de manera notable los posteriores análisis espaciales.

Muchos han sido los detractores del análisis de los suelos de ocupación para yacimientos excavados entre la segunda mitad del s. XX y la década de 
los 90, ya que se considera que en los procesos de recuperación de datos no constituía una premisa indispensable la búsqueda de suelos de ocupación. Sin embargo, consideramos que la presencia o ausencia de elementos indicativos de la existencia de suelos de ocupación o del desarrollo de labores concretas es independiente de la voluntad del excavador. Así pues, tecnologías tales como los Sistemas de Información Geográfica (SIG), apoyadas por un trabajo de campo de cierta calidad, hacen que se puedan reconstruir los registros con tal precisión que nos permitan la identificación de estructuras latentes en los suelos, como en el momento de ser excavados.

\section{DIFERENCIAS EN EL PROCESADO DE LOS DATOS}

El carácter espacial de los datos procesados en nuestro estudio hace necesario el empleo de herramientas como los Sistemas de Información Geográfica. Estos programas informáticos posibilitan gestionar por un lado, datos cuantitativos de acumulación y/o dispersión en forma de varianzas, etc. representados en ejes de coordenadas cartesianas y, por otro, todos aquellos datos cualitativos en forma de atributos o categorías, en los que se especifican todos los datos fruto de los análisis tecnológicos, tipológicos o funcionales. Por todo ello, estos sistemas informáticos nos posibilitan tener una visión dinámica, más allá de los mapas de puntos conocidos, ya que demuestran gran versatilidad en el tratamiento de la información, que varía en función de las preguntas que queramos responder en cada momento.

Los Sistemas de Información Geográfica no sólo permiten gestionar información de muy distinta índole sino que permite procesar datos y crear otros nuevos a raíz de los análisis estadísticos y espaciales integrados en el sistema.

En el propio proceso de excavación, sobre todo en aquellas excavaciones llevadas a cabo en la segunda mitad del s. XX, existen diferencias en los procesos de recuperación de información, que también varían en función de los avances tecnológicos disponibles y el valor atribuido a cada tipo de dato recuperado. Así pues la precisión de recuperación y posicionamiento de las piezas dependen del criterio subjetivo del propio excavador.

La diferente naturaleza de cada uno de los datos que se recogen en la excavación arqueológica y las diferencias en el procesado posterior hacen que nos veamos en la obligación de procesar cada tipo de datos que nos llegan según la calidad de los mismos y el tipo de información potencial de cada uno. Así derivado de los procesos de excavación realizados en la segunda mitad del s. XX podemos distinguir entre los datos que poseen coordenadas y aquellos que poseen como única referencia espacial su pertenencia a un subcuadro, o sector, que normalmente posee una extensión de $33 \mathrm{~cm}$. de lado. 
El análisis de los datos que poseen coordenadas exactas se realiza a través de un gráfico de puntos. Se trata de un mapa de carácter vectorial en el que cada dato se representa por medio de un punto posicionado dentro del plano general del yacimiento en el lugar exacto donde se ha encontrado. Los SIG favorecen tener una visión dinámica de la información por lo que la representación de cada punto variará cualitativamente. Se crea así un gráfico espacial en el que se representan una serie de datos cualitativos en el espacio físico. Son los datos no espaciales, es decir, todos aquellos datos derivados de los análisis tanto tecnológicos, tipológicos o funcionales, a modo de atributos, los que caracterizan la representación de los datos que se visualizan de manera espacial.

Más adelante, serán los pertinentes estudios funcionales los que nos permitirán de manera más fiable establecer relaciones entre los distintos tipos de utillaje, a la vez que la identificación de actividades latentes en los suelos de habitación. Sin embargo, los estudios tecnológicos de cadenas operativas aportan también información relevante acerca de la posibilidad de identificar áreas de actividades concretas.

La representación espacial de los elementos coordenados favorecerá la realización de análisis estadísticos tales como los análisis de densidad Kernel, lo que permitirá crear mapas de densidades favoreciendo la comparación de los resultados con la distribución de otro tipo de materiales.

Sólo a través del estudio conjunto del registro arqueológico, mediante relaciones directas entre materiales, independientemente de su naturaleza o uso, se podrán inferir áreas de actividades $\operatorname{concretas}^{16}$. En esta línea también se realizan estudios como el análisis del vecino más próximo, la clasificación según coordenadas de Kintingh y Ammerman ${ }^{17}$, el análisis de la densidad de Johnson $^{18}$ y unconstrained clustering de Whallon ${ }^{19}$.

Aquellos datos que, debido a los procesos de excavación, sólo conservan como única referencia espacial el sector o subcuadro, tomarán las coordenadas del centroide del mismo para análisis de los elementos concretos.

Tomamos el centroide como coordenadas de cada objeto de interés, susceptible de ser estudiado individualmente, ya que entendemos que el rango de error para aquellos elementos distribuidos en toda la superficie del sector que mide $33,3 \mathrm{~cm}$. de lado, es aceptable para la escala de análisis que estamos realizando.

En los procesos que requieran un análisis cuantitativo y estadístico, es requisito imprescindible representar mediante porcentajes el binomio presencia-ausencia o la acumulación de elementos. Para ello se procesarán a través de un mapa vectorial en el que los datos cuantitativos se asociarán al polígono que representa el sector correspondiente, asignando a cada uno una gradación de color. 
Del mismo modo, los datos sin referencias espaciales exactas se pueden procesar en forma de mapas de densidades (dot density), como los restos faunísticos, que se procesarán en función de los taxones, índices de fracturación, número mínimo de individuos y marcas de utilización. Como ya habíamos mencionado, la gran versatilidad en las representaciones espaciales de los Sistemas de Información Geográfica nos permite responder a numerosas cuestiones que los patrones habitacionales aun nos suscitan como el reconocimiento de zonas especializadas de despiece, así como la disponibilidad de recursos del grupo que habitaba la cueva para ese momento concreto.

De manera similar al caso de los restos faunísticos, los restos y desechos del proceso de talla se analizarán en función de la dispersión, tamaños, etc., así podremos inferir zonas de talla, de descortezado de la materia prima, etc., que del mismo modo se pueden relacionar con posibles remontajes ${ }^{20}$.

El empleo de los SIG nos permite tener una visión de la distribución del espacio en los lugares de habitación no sólo sincrónica, en función de los niveles a estudiar sino también diacrónica en la que se identifique el uso reiterado en el tiempo de una zona destinada a una labor concreta. Así pues estas herramientas nos facilitan la comparación de los datos de forma diacrónica a través del sistema de capas.

Movimientos Deposicionales ESTUdiAdos POR LOS Sistemas DE INFORMACIÓN GEOGRÁFICA.

Los niveles arqueológicos son susceptibles de sufrir numerosos procesos, biológicos, químicos y geomorfológicos que pueden afectar a la propia conformación de los mismos. Como ya hemos mencionado anteriormente, la conservación del registro de las zonas de hábitat en cueva es muy irregular, pudiéndose encontrar niveles que han sufrido remociones de tierra, inundaciones o alteraciones biológicas.

Tener en cuenta estos procesos nos obligará a estudiar los posibles movimientos deposicionales, ya que muchos de ellos alteran los suelos de ocupación hasta el punto de trasladar los materiales de su posición original en el momento que fueron depositados, lo que, evidentemente, invalida de alguna manera dicho registro para una identificación de los patrones habitacionales, pues la realidad de los datos es alterada, produciendo un 'ruido' que no podría ser asumido para una interpretación posterior.

No obstante, las herramientas del SIG, especialmente las posibilidades que nos plantean las aplicaciones de 3D y las técnicas del modelado del terreno, permiten que podamos reconstruir, de alguna manera, los procesos 
deposicionales, ya que nos da la posibilidad de reconstruir y procesar cada capa de excavación de manera aislada, consiguiendo recrear las dinámicas de formación y especialmente las de modificación de los niveles de ocupación

Para este análisis recurrimos a datos de cotas iniciales y finales de cada capa que se asocian en una tabla a los propios elementos coordenados, creando así una malla irregular que se representará a través escalas de progresión representada con una red de triángulos irregulares o TIN (Triangulated Irregular Network). Esta red nos posibilita crear un modelo digital del terreno a nivel microespacial, permitiéndonos realizar análisis como la estimación de vías óptimas o análisis de visibilidades. Así se pretende aplicar el análisis que habitualmente se usa en los estudios macroespaciales a una escala menor con el fin de responder a cuestiones de procesos deposicionales ${ }^{21}$.

\section{CONCLUSIONES}

En este artículo se ha intentado hacer una aproximación metodológica al uso de los Sistemas del Información Geográfica y al análisis de yacimientos paleolíticos de manera que se analizan las posibilidades que estas herramientas ofrecen para el estudio de los suelos de ocupación y, por ende, al uso del espacio de habitación durante el Paleolítico Superior, superando problemáticas como los procesos deposicionales.

Los SIG han sido herramientas empleadas de forma general para la realización de estudios macroespaciales (inter-sites) en los que primaban estudios tales como análisis predictivos de localización de yacimientos, en los que intervienen parámetros como visibilidad, insolación o accesibilidad. Sin embargo, pocos son los estudios que emplean estas herramientas para los análisis microespaciales (intra-sites), lo que ha favorecido un pobre y variable desarrollo metodológico, que, sin duda, debe avanzar en estudios futuros. Entendemos como necesario progresar en esta línea de investigación, aplicando las nuevas tecnologías a nuestro alcance.

El espacio social de los grupos paleolíticos debe entenderse en términos de relaciones espaciales, tanto a niveles macroespaciales como microespaciales. Centrándonos en el plano microespacial, tema principal de este artículo, se ha recalcado la necesidad de realizar un estudio integral del conjunto de los materiales depositados en forma de suelos de ocupación, lo que permite el análisis y teorización sobre el uso del espacio paleolítico en el lugar de habitación.

\section{BIBLIOGRAFÍA PRINCIPAL}


BORDES, F. (1975): "Sur la notion de sol d'habitat en préhistoire paléolithique" Bulletin de la Société Préhistorique Française 72 (5), pp. 139-144.

CLARKE, D.L. (1977): “ Spatial Information in Archaeology ". En: Clarke, D.L. (Ed.), Spatial Archaeology. Academic Press, London, pp. 1-32.

CONOLLY, J. \& LAKE, M. (2009): Sistemas de Información geográfica aplicados a la arqueología. Bellaterra Arqueología, Barcelona.

DJINDJIAN, F. (1991): Méthodes pour l'archéologie, Armand Colin, Paris.

DJINDJIAN, F. (1999) : "L'analyse spatiale de l'habitat : un état de l'art ". Archeologia e Calcolatori, 10, pp. 17-32.

GRAU MIRA, I. (2006): La aplicación de los SIG en la arqueología del paisaje. Lucentum. Anejo; 15 Serie arqueológica, Universidad de Alicante.

KEELER, D. (2007): "Intrasite spatial análisis of a Late Upper Paleolithic french site using geographic information systems". Journal of World Anthropology: Occasional Papers vol III, n I, pp 29-33.

LEROI GOURHAN, A. (1975): "L’hábitat au Paléolithique Supérieur" UISPP, IXe congrès, Colloque XIII (Nice, 15 septembre). CNRS, Paris, pp. 85-92.

ONTAÑON PEREDO, R. (2003): "Sols et structures d'habitat du Paléolithique supérieur, nouvelles données depuis les Cantabres: La Galerie Inférieure de La Garma (Cantabria, Espagne)". L'Anthropologie, 107/3, pp. 333-363.

YAR, B y DUBOIS, P (1999): Les structures d'habitat au Paléolithique en France. Ed. Monique mergoil, Montagnac.

WHEATLEY, DAVID (2002): Spatial technology and archaeology : the archaeological applications of GIS. Taylor \& Francis, Publicac London.

\section{NOTAS}

* Becaria de la Fundación de Patrimonio Histórico de Castilla y León.

${ }^{1}$ SANZ, N. (1993): "Para una lógica social del espacio en Prehistoria", Complutum 4, p. 242.

${ }^{2}$ CONOLLY, J. \& LAKE, M. (2009): Sistemas de Información geográfica aplicados a la arqueología. Bellaterra Arqueología, Barcelona pp:18-19

${ }^{3}$ SANZ, N. (1993): "Para una lógica social del espacio en Prehistoria”, Complutum 4, pp. 242-245.

${ }^{4}$ LEROI-GOURHAN, A. (1975): “L’hábitat au Paléolithique Supérieur” UISPP, IXe congrès, Colloque XIII (Nice, 15 septembre). CNRS, Paris, pp. 85-92.

${ }^{5}$ RIGAUD, J. (1976) : «Les structures d'habitat d'un niveau de Périgordien Supèrieur du 
Flageolet I (Bézenac, Dordogne) ». UISPP, IXe congrès, Colloque XIII (Nice, 15 septembre). CNRS, Paris, pp. 94

${ }^{6}$ DJINDJIAN, F. (1991): Méthodes pour l'archéologie, Armand Colin, Paris

${ }^{7}$ PEYRONY, D. (1932): "Les gisements préhistoriques de Bourdelles (Dordogne) ". Archives de l'Institut de Paléontologie Humaine. Mém 10. Masson et Cie, éditeurs, Paris

${ }^{8}$ EFIMENKO, P. P. (1958): “Kostenki, I.” Akademia Nauk SSSR. Institut istorii materialnoj kultury, Moskava-Leningrad.

${ }^{9}$ BORDES, F. (1975): "Sur la notion de sol d'habitat en préhistoire paléolithique" Bulletin de la Société Prébistorique Française 72 (5), pp. :139.

${ }^{10}$ LEROI GOURHAN, A. y BRÉZILLON, M. (1972): Fouilles de Pincevent. Essai d'analyse ethnographique d'un habitat magdalénien. Éditions du CNRS, Paris (Suppléments à Galia Préhistoire VII) pp. :246-256.

${ }^{11}$ BINFORD, L.R. (1983) : In pursuit of the past: decoding the Analogues record. Thames and Hudson, London.

${ }^{12}$ CLARKE, D.L. (1977): “ Spatial Information in Archaeology ”. En: Clarke, D.L. (Ed.), Spatial Archaeology. Academic Press, London, pp. 1-32.

${ }^{13}$ HODDER, I. \& ORTON, C. (1976): Spatial Analysis in Archaeology Cambridge University Press, Cambridge.

${ }^{14}$ ONTAÑON PEREDO, R. (2003): "Sols et structures d’habitat du Paléolithique supérieur, nouvelles données depuis les Cantabres: La Galerie Inférieure de La Garma (Cantabria, Espagne)" L'Anthropologie 107/3: 333-363.

${ }^{15}$ YAR, B. y DUBOIS, P. (1999): Les structures d'habitat au Paléolithique en France. Ed Monique mergoil, Montagnac.

${ }^{16}$ KEELER, D. (2007): "Intrasite spatial análisis of a Late Upper Paleolithic french site using geographic information systems". Journal of World Anthropology: Occasional Papers vol III, n I pp 29-33.

${ }^{17}$ KINTIGH, K.W., AMMERMAN, A.J. (1982): "Heuristic Approaches to Spatial Analysis in archaeology”. American Antiquity 47 (1), pp. 31-63.

${ }^{18}$ JOHNSON, I. (1984): “ Cell frequency recording and analysis or artifact distributions". En: HIETALA, H.J. (Ed.): Intrasite spatial analysis in archaeology. Cambridge University Press, NewYork, pp. 75-96.

${ }^{19}$ WHALLON, R. (1984): "Unconstrained clustering for the analysis of spatial distributions in archaeology" In: HIETALA, H.J. (Ed.): Intrasite spatial analysis in archaeology. Cambridge University Press, New York, pp. 242-277.

${ }^{20}$ WHEATLEY, DAVID (2002): Spatial technology and archaeology : the archaeological applications of GIS. Taylor \& Francis, Publicac, London. 
${ }^{21}$ NIGRO, J. D.; UNGAR, P. S.; RUITERAND, D. J. y BERGER, L. R. (2003): “Developing a Geogtaphic Information System (GIS) for zapping and analysing fósil deposits at Swartkrans, Gauteng Province, South Africa" Journal of Archaeological Science, 30, p 317-324. 